\title{
EDITORIAL
}

\section{Congratulations to 2016 EST AWARD WinNERS}

BJS Essential Surgical Techniques (EST) has been proud to offer awards for the best Subspecialty Procedures (SP) article and Key Procedures (KP) video published each year, and it is our pleasure to congratulate the 2016 winners: Austin T. Fragomen and S. Robert Rozbruch for their SP article "Lengthening of the Femur with a Remote-Controlled Magnetic Intramedullary Nail: Retrograde Technique" (2016 May 11;6[2]:e20) and Jesse D. Chlebeck, Christopher E. Birch, and Jennifer W. Lisle for their KP video "Percutaneous in Situ Fixation of Slipped Capital Femoral Epiphysis" (2016 Mar 23;6[1]:e11). Their usage of the multimedia format to educate surgeons in an elegant and succinct manner is admirable.

Keeping abreast of both new procedures and technical improvements in established procedures is challenging as our specialty continues to expand with new knowledge disseminated in a multitude of formats. JBJS EST shines a light solely on those procedures whose outcomes have been established in peerreviewed journals so that surgeons can use their time most efficiently by learning new techniques that are most likely to stand the test of time. We offer both our Subspecialty Procedures series, concentrating on operations that are most often performed by subspecialists, and our Key Procedures series, based on fundamental operations that nearly all orthopaedists perform. Both series use video and an outline format to educate as efficiently as possible. We encourage readers to peruse these award-winning articles and discover how JBJS $E S T$ is changing the way that we learn by providing valuable, practical content that surgeons can access immediately when it is most important - at the point of care- to provide optimal treatment for their patients.

We are also happy to announce that we will again be offering awards for the best JBJS EST articles and videos in 2017.

Edward Y. Cheng, MD 